\title{
Association between Maternal Dietary Diversity and Neonatal Birth Size
}

\section{ISSN: 2637-8078}

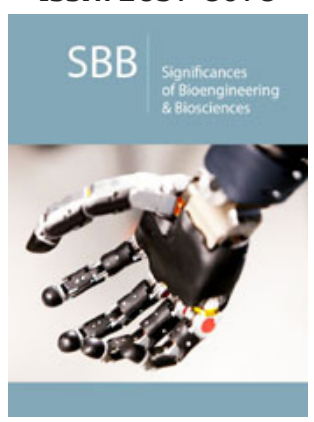

*Corresponding author: Sana Noreen, Institute of Diet and Nutritional Sciences, Faculty of Allied Health Sciences, The University of Lahore, Lahore, Pakistan

Submission: 侮 September 24, 2019

Published: 笽October 09, 2019

Volume 3 - Issue 5

How to cite this article: Sana Noreen, Ayesha S, Imran M, Gilani S, Ahsan F, et al. Association between Maternal Dietary Diversity and Neonatal Birth Size. Significances Bioeng Biosci.3(5). SBB.000572.2019.

DOI: 10.31031/SBB.2019.03.000572

Copyright@ Sana Noreen, This article is distributed under the terms of the Creative Commons Attribution 4.0 International License, which permits unrestricted use and redistribution provided that the original author and source are credited.

\author{
Sana Noreen*, Ayesha S, Imran M, Gilani SA, Ahsan F, Sikander S, Raza A, \\ Basharat S, and Jawad FA \\ Institute of Diet and Nutritional Sciences, Faculty of Allied Health Sciences, The University of \\ Lahore, Lahore, Pakistan
}

\begin{abstract}
Background: Maternal under-nutrition is one of the major causes of maternal morbidity and mortality, particularly in the developing countries. Maternal nutrition has direct association with neonatal birth size. It is estimated that $15 \%$ to $20 \%$ of all births worldwide are Low Birth Weight (LBW) representing more than 20 million births a year.
\end{abstract}

Method: In this study cross-sectional research design was used to survey 385 pregnant women who were selected purposively from one public and four private Lahore, Pakistan. Participants aged 25-30 years, were enrolled after taking written informed consent. Socio-demographics profiles of the participants including maternal/paternal age and education was recorded through a structured questionnaire. Maternal anthropometric measurements such as weight, height, Body Mass Index (BMI), Mid- Upper Arm Circumference (MUAC) and neonatal weight, length, head and chest circumference were measured. Maternal dietary intake was assessed by 24 Hour Recall and Food Frequency Questionnaire (FFQ). Dietary Diversity Score (DDS) was calculated from three days 24 Hour Recall performed. Data was collected from Feb-July 2017. Descriptive statistics were calculated for all the variables. Chi-square test was applied to observe the association between independent and dependent variables. Results were analyzed by using IBM Statistical Package for Social Sciences (SPSS) version 23. The p-value less than 0.05 was taken as significant.

Result: The mean of maternal age was $27.92( \pm 4.033)$ years and the mean of neonatal birth weight was $3.026( \pm 0.369) \mathrm{kg}$, length $48.64( \pm 3.543) \mathrm{cm}$, head circumference $32.46( \pm 1.9747) \mathrm{cm}$ and chest circumference $31.485( \pm 1.947) \mathrm{cm}$. Head circumference of the neonate was significantly associated with dietary diversity ( $\mathrm{p}$-value 0.001 ) whereas neonatal chest circumference, length and birth weight was not associated with dietary diversity ( $\mathrm{p}$-value $>0.05$ ).

Conclusion: It was concluded that increased maternal education, paternal age, paternal education and income resulted in high dietary diversity. Furthermore, dietary diversity was found to be associated with head circumference of the neonates, while dietary diversity was not linked with other neonatal characteristics.

Keywords: Food intake; Pregnancy; Dietary diversity scores (DDS); Low birth weight (LBW); Birth size

\section{Introduction}

Around $16 \%$ of all newborn babies of the world which make a scientific number of 22 million were underweight. South Asia has the highest prevalence of underweight babies in the world, with one in four newborns weighing less than 2,500 grams [1]. Whereas, $12-25 \%$ of newborn babies are underweight in Pakistan [2,3]. Low birth weight is a significant predictor of prenatal mortality as well as morbidity [4]. Low birth weight increases the risk of diabetes, heart diseases and cancer [5]. Weight of the baby at birth is the outcome of maternal health and nutrition. Undernourishment within womb increases the chances of early death [6]. The survivors tend to have weak immune system and increased risk of disease. They have the probability to remain undernourished resulting in weak muscles and low mental abilities [7]. Therefore, it is inferred that maternal nutrition plays a major role in affecting the growth of fetus and birth outcomes. Studies have shown that insufficient consumption of important nutrients and less intake of energy during pregnancy will have detrimental health effects on adulthood [8]. Studies indicate that food taken during pregnancy has impending effects on 
birth outcomes [9]. Women belonging to developing countries can have deficiency of many micronutrients, such as iron, folic acid, iodine, zinc, vitamin A and D [10] when they reach reproductive age especially during pregnancy [11]. Malnourishment during pregnancy can be due to poverty, poor diet and high fertility rate [12]. Evidence suggests that increase in age and education also linked with healthy and diverse consumption of diet by pregnant women.

It is also suggested that women with high socio-economic status are more probable to have healthier food habits and better prospects to maintain the dietary records that are reliable with nutritional recommendations or dietary guidelines. Whereas women who have less education and have socio-demographic features like non-working, increased parity, are more liable to have unhealthy and non-diverse diet [13]. The dietary diversity questionnaire is a quick, comprehensive, easy to use and monitor cost effective tool. Internationally, it has been commended for diversifying the foods across and within food groups [14]. A dietary habit are mostly prompted by tradition and by the food accessible for intake, studying the food constituent of food inside a study population may lay foundation for prospective inferences for better maternal and infant nutrition [15]. Only birth weight has been extensively invested but other parameters of anthropometric measurements like head circumference, chest circumferences can provide useful information regarding the health of neonates [16,17]. The aim of the study was to assess the relationship between maternal dietary diversity scores and neonatal birth size among women attending tertiary care government hospitals in Lahore, Pakistan.

\section{Material and Methods}

\section{Study design $\&$ settings}

This is cross-sectional research design. Ethical approval by guarantying no potential harm \& associated risk and non-disclosure of data was obtained from Medical Superintendent of Tertiary Care Government Hospitals, Lahore, Pakistan.

\section{Sample size}

Three hundred and eighty-five participants were selected purposively from public and private hospitals. Participants aged 25-30 years, enrolled consecutively after taking written informed consent. The inclusion criteria consisted of including pregnant women that were registered for twenty or more weeks with the hospital while still birth, multiple births, and neonates with congenital abnormalities was excluded. The data was collected in the period of six months from Feb-July 2017.

\section{Demographic data}

A structured questionnaire was used to collect data on demographic and socio-economic factors such as age, education, parity, physical activity as well as antenatal records was used in the study [18]. The structured questionnaire was administered face to face.

\section{Anthropometric measurements}

Maternal anthropometric measurement (weight, height, BMI, MUAC) and neonatal weight, length, head and chest circumference was measured to assess neonatal birth size. Weight of the mother was measured in kilograms with weighing scale (West point weight scale, Japan) [19] and rounded off to nearest $0.1 \mathrm{~kg}$. Height of the mother was measured with stadiometer (Seca 216-the mechanical height meter, Seca Corp., Ukraine, Kiev), without shoes and rounded off to nearest $0.1 \mathrm{~cm}$. Maternal Body Mass Index (BMI) or Quetelet index was calculated by dividing weight $(\mathrm{kg})$ by the square of height (cm) [20]. Maternal mid upper-arm circumference (MUAC) was measured by non-stretchable measuring tape [21]. Tape was fixed at the mid-point between the elbow and the shoulder (acromion and olecranon) and place the tape measure around the left arm (the arm should be relaxed and hang down the side of the body). Weight of the baby at birth was measured in kilograms by infant pan scale (Lacia, BF 2051). Length of the baby was measured in centimeters by using an infant-o-meter (Baby Length Measuring MAT-SECA 210). Occipital- frontal head circumference of the baby was measured in centimeters by wrapping the measuring tape around the widest possible circumference - from the forehead (often 1-2 fingers above the eyebrow) around to the widest part of the back of the head. Chest circumference was also measured in centimeters with non-stretchable measuring tape, measuring the circumference of the chest at the level of the nipples during normal breathing [22]. All measurements were taken three times and average was calculated to ensure reliability.

\section{Maternal dietary intake}

Dietary intake was assessed by 24 Hour Recall and Food Frequency Questionnaire (FFQ). It based on a structured interview to internment detailed information about all foods and beverages taken by the mothers (respondent) [23]. Dietary assessment was carried out in a face-to-face interview with pregnant women participating in the study, using a Food Frequency Questionnaire (FFQ) to get information on consumption of inquired foods and beverages over the specified period [24]. FFQ developed by Mikolajczyk [25], was used in the current study. Dietary Diversity Scores (DDS) was based on three days 24-hours recall. It usually measured by summing the number of foods or food groups consumed over a reference period [26]. The reference period usually ranges from one to three days, but seven days is also often used. A score of 1 was given to a food group that consumed at least once in the past three days. 0 was given to food groups that was not consumed even once in the past three days. The sum of all scores was divided by three to get an average dietary diversity score for each individual for ease of analysis. Dietary diversity scores have been previously validated $[27,28]$.

\section{Data analysis}

Descriptive statistics was calculated for all variables. Score less than 4.9 was categorized as low score. 5-7.9 was categorized as medium and score more than 8 as high. Anova was applied to 
compare socio-demographic characteristic with categories of Dietary Diversity. Chi-square was applied to observe the association between independent and dependent variables. A p-value less than 0.05 were taken as significant and data was analyzed using SPSS v.23.

\section{Result}

Table 1 showed the descriptive statistics characteristic of the participants. The frequency from Services Hospital 187(48.6\%), National Hospital 69(17.9\%), Naaz Hospital 66(17.1\%), Prime Care Hospital 56(14.6\%) and Aadil Hospital 7(1.8\%), correspondingly. Analysis of data of the participants showed that $26 \%$ women were doing jobs and 73\% were un-employed. 91.2\% women belonged to Punjab province while $8.8 \%$ belonged to other provinces.
91.7\% women were Muslims whereas $8.3 \%$ women belonged to other religions. 93.5\% participants were from Lahore and $6.5 \%$ were from other cities, $60.8 \%$ participants preferred joint family system whereas $39.2 \%$ were living in independent family system. $34 \%$ women had servants for help while $66 \%$ women were doing their home task by themselves. It was also revealed that husbands of $3.6 \%$ participants were doing government job, $34 \%$ had selfbusiness, $61.6 \%$ were doing private jobs and $0.8 \%$ did not have any job. Table 2 showed the outcome of socio demographic profile of the participants of this study. The mean of maternal age was $27.9( \pm 4.033)$ years, maternal education was $10.68( \pm 4.902)$ years, paternal age was $31.22( \pm 4.439)$ years, paternal education was $12.20( \pm 3.725)$ and income was $52,195.31( \pm 43902.585)$ PKR, respectively.

Table 1: Descriptive statistics characteristic of the participants.

\begin{tabular}{|c|c|c|c|}
\hline & & Frequency & Percentage \\
\hline \multirow{5}{*}{ Hospital } & Services Hospital & 187 & 48.6 \\
\hline & National Hospital & 69 & 17.9 \\
\hline & Naaz Hospital & 66 & 17.1 \\
\hline & Prime Care Hospital & 56 & 14.6 \\
\hline & Aadil Hospital & 7 & 1.8 \\
\hline \multirow{2}{*}{ Maternal employment } & Yes & 101 & 26.2 \\
\hline & No & 284 & 73.8 \\
\hline \multirow{2}{*}{ Ethnicity } & Punjabi & 351 & 91.2 \\
\hline & Non-Punjabi & 34 & 8.8 \\
\hline \multirow{2}{*}{ Religion } & Muslims & 353 & 91.7 \\
\hline & Non-Muslims & 32 & 8.3 \\
\hline \multirow{2}{*}{ City } & Lahore & 360 & 93.5 \\
\hline & Other city & 25 & 6.5 \\
\hline \multirow{2}{*}{ Joint family system } & Yes & 234 & 60.8 \\
\hline & No & 151 & 39.2 \\
\hline \multirow{2}{*}{ Servants } & Yes & 131 & 34 \\
\hline & No & 254 & 66 \\
\hline \multirow{4}{*}{ Husband occupation } & Jobless & 3 & 0.8 \\
\hline & Private job & 237 & 61.6 \\
\hline & Government job & 14 & 3.6 \\
\hline & Self-business & 131 & 34 \\
\hline
\end{tabular}

Table 2: Socio demographic profile of participants.

\begin{tabular}{|c|c|c|}
\hline & Mean & Std. Deviation \pm \\
\hline Maternal Age (yrs) & 27.92 & 4.033 \\
\hline Maternal Education (yrs) & 10.68 & 4.902 \\
\hline Paternal Age (yrs) & 31.22 & 3.439 \\
\hline Paternal Education (yrs) & 12.2 & 43903 \\
\hline Income (PKR (yrs) & 52195 & 425 \\
\hline
\end{tabular}


Table 3 showed the comparison of sociodemographic characteristic of participants. The assessment of the results concluded that there was no significant difference found with maternal age $(p=0.063)$ whereas statistical difference was present with maternal education $(p=0.001)$, paternal age $(p=0.014)$, paternal education $(p=0.006)$ and income $(p=0.021)$. It was also concluded that increased maternal education $12.63( \pm 3.995)$ years, paternal age $31.36( \pm 4.706)$ years, paternal education 13.34( \pm 2.830$)$ years and income $62,888.89( \pm 40624.892)$ PKR resulted in high dietary diversity. Table 4 showed maternal anthropometric measurements characteristics. Means of maternal anthropometric measurements with dietary diversity scores had been evaluated to find their significance and the results concluded that increased maternal booking weight BMI $25.2( \pm 3.222) \mathrm{kg} / \mathrm{m}^{2}$, pre-delivery BMI $29.6( \pm 3.101) \mathrm{kg} / \mathrm{m}^{2}$, and postpartum BMI 27.5 $( \pm 3.230) \mathrm{kg} / \mathrm{m}^{2}$, resulted in high dietary diversity. Table 5 presented the association between dietary diversity and anthropometric measurement of neonates and the results showed that neonatal birth weight, length, chest circumference were not found to be associated with dietary diversity as their significance values were high but the head circumference was found associated with dietary diversity as significant value was $17.178(\mathrm{p}=0.001) \mathrm{cm}$.

Table 3: Comparison of socio demographic profile of participants with dietary diversity.

\begin{tabular}{|c|c|c|c|c|c|}
\hline & $<4.9$ (Low) & 5-7.9 (Medium) & $>8$ (High) & $\mathbf{F}$ & P-value \\
\hline Maternal Age (years) SD \pm & $25(3.432)$ & $27.95(4.154)$ & $28.16(3.448)$ & 2.791 & 0.063 \\
\hline $\begin{array}{l}\text { Maternal Education (grades) } \\
\text { SD } \pm\end{array}$ & $9.10(2.846)$ & $10.25(5.040)$ & $12.63(3.995)$ & 7.691 & $.001^{*}$ \\
\hline Paternal Age(years) SD \pm & $27(3.615)$ & $31.32(4.348)$ & $31.36(4.706)$ & 4.285 & $.014^{*}$ \\
\hline $\begin{array}{l}\text { Paternal Education (grades) } \\
\text { SD } \pm\end{array}$ & $10.40(4.195)$ & $11.99(3.846)$ & $13.34(2.830)$ & 5.207 & $.006^{*}$ \\
\hline $\begin{array}{l}\text { Paternal Income (rupees) } \\
\text { SD } \pm\end{array}$ & $28400(26592.397)$ & $50433.77(44640.840)$ & $62888.89(40624.892)$ & 3.906 & $.021^{*}$ \\
\hline
\end{tabular}

Table 4: Comparison of maternal anthropometric measurements with dietary diversity.

\begin{tabular}{|c|c|c|c|c|c|}
\hline Booking weight (lbs) SD \pm & $137.1(22.268)$ & $135.2(18.925)$ & $142.6(19.357)$ & 4.378 & $.013^{*}$ \\
\hline Pre-delivery weight (lbs) SD \pm & $156.8(23.476)$ & $158.6(19.936)$ & $\begin{array}{c}167.5 \\
(-19.377)\end{array}$ & 6.076 & $.003^{*}$ \\
\hline Post-partum weight (lbs) SD \pm & $143.9(19.981)$ & $146.0(19.861)$ & $\begin{array}{c}154.6 \\
(-19.155)\end{array}$ & 5.822 & $.003^{*}$ \\
\hline BMI.1 $\left(\mathrm{kg} / \mathrm{m}^{2}\right) \mathrm{SD} \pm$ & $24.9(3.657)$ & $24.9(11.907)$ & $\begin{array}{c}25.2 \\
(-3.222)\end{array}$ & 0.023 & 0.977 \\
\hline BMI.2 (kg $\left./ \mathrm{m}^{2}\right) \mathrm{SD} \pm$ & $28.5(3.664)$ & $28.4(3.411)$ & $\begin{array}{c}29.6 \\
(-3.101)\end{array}$ & 3.759 & $.024^{*}$ \\
\hline BMI.3 $\left(\mathrm{kg} / \mathrm{m}^{2}\right) \mathrm{SD} \pm$ & $26.2(3.282)$ & $26.3(4.371)$ & $27.5(3.230)$ & 2.395 & 0.092 \\
\hline Maternal height $(\mathrm{cm}) \mathrm{SD} \pm$ & $157.9(4.606)$ & $158.9(4.569)$ & $160.3(4.615)$ & 3.024 & $.050^{*}$ \\
\hline MUAC $(\mathrm{cm}) \mathrm{SD} \pm$ & $29.1(3.104)$ & $25.7(3.772)$ & $26.7(4.475)$ & 5.195 & $.006^{*}$ \\
\hline Weight gain (lbs) SD \pm & $20.3(6.343)$ & $23.2(7.726)$ & $24.9(7.327)$ & 2.362 & 0.096 \\
\hline
\end{tabular}

Table 5: Association between dietary diversity and anthropometric measurement of neonates.

\begin{tabular}{|c|c|c|c|c|c|c|}
\hline & & Low & Medium & High & Chi-square & Significance (2-sided) \\
\hline \multirow{2}{*}{ Birth weight (kg) } & 2.49 & 0 & 14 & 2 & \multirow{2}{*}{0.976} & \multirow{2}{*}{0.614} \\
\hline & $>2.5$ & 10 & 288 & 71 & & \\
\hline \multirow{2}{*}{ Length $(\mathrm{cm})$} & $<45$ & 3 & 37 & 6 & \multirow{2}{*}{4.0829} & \multirow{2}{*}{0.129} \\
\hline & $>46$ & 7 & 265 & 67 & & \\
\hline \multirow{2}{*}{ Head circumference $(\mathrm{cm})$} & $<32$ & 8 & 119 & 15 & \multirow{2}{*}{17.178} & \multirow{2}{*}{$.001^{*}$} \\
\hline & $>32.5$ & 2 & 183 & 58 & & \\
\hline \multirow{2}{*}{ Chest circumference (cm) } & $<32$ & 5 & 212 & 46 & \multirow{2}{*}{2.992} & \multirow{2}{*}{0.224} \\
\hline & $>32.5$ & 5 & 90 & 27 & & \\
\hline
\end{tabular}




\section{Discussion}

The results of presentstudy indicated that the sociodemographic status of the participants like maternal education, paternal age, education and paternal income was associated with the dietary diversity score of the participants. It was clear from the present study that maternal and paternal age and education with household income was a major determinant of maternal dietary diversity [29]. Maternal dietary diversity scores were sufficiently high in women of higher socioeconomic class. By using Food Frequency Questionnaire, it was found that all the participants were consuming cereal several times a day, $79.9 \%$ participants were consuming milk and $51.4 \%$ were taking fruits on daily basis. $54.5 \%$ meat, $54.3 \%$ vegetables, $37.1 \%$ sweets were taken several times/weeks. $42.6 \%$ fast food, $37.7 \%$ soft drinks, $41.6 \%$ cookies and $36.1 \%$ raw salad were taken 1-4 times/month by the participants, respectively. Previous studies have been conducted in Malaysia to check the association between consumption of fruits and vegetables and birth size and it revealed significant association. This suggested that existence of phytochemicals in foods may play an important role in birth size and as the frequency of intake of fruits and vegetables is high in participants of current study, so chances of squeezed birth size of neonate are significantly low [30]. In the current study, mean of neonatal weight was near $3 \mathrm{~kg}$, length $48.64 \mathrm{~cm}$, head and chest circumference were $32.46 \mathrm{~cm}$ and $31.48 \mathrm{~cm}$. No association could be found between dietary diversity and birth weight, length and chest circumference of the newborn baby. The results are in contrast to previous studies done in Ethiopia in 2016 and Ghana where inadequate dietary diversity resulted in a higher risk of low birth weight. It is noticed that they did not measure other parameters of birth size [31]. The results indicated that dietary diversity score had statistically significant association with head circumference of the neonates [32]. No studies could be found in favor or against this result as among anthropometric measurements only birth weight has been extensively studied. Measuring head circumference (HC) in infants is a quick, simple, noninvasive and reliable procedure for determining underlying brain size. Head growth in early neonatal life is very important. Avoiding growth impairment during neonatal care may allow for optimal cortical development. After plotting the measurement on sex and age-appropriate charts, family physicians can determine if HC is within normal limits, too large (megacephaly), or too small (microcephaly). Serial head circumference measurements during well-child visits are critical to screening young children for possible cognitive or motor delays. Early detection and timely intervention could reduce neonatal morbidity, mortality and decreases the health care costs [33].

\section{Conclusion}

It was concluded that dietary diversity was associated with head circumference of the neonates, while dietary diversity was not associated with other neonatal characteristics (like weight, length and chest circumference). Increased maternal education, paternal age, paternal education and paternal income resulted in high dietary diversity.

\section{References}

1. Wardlaw T, You D, Hug L, Amouzou A, Newby H (2014) UNICEF report: Enormous progress in child survival but greater focus on newborns urgently needed. Reprod Health 11: 82.

2. Khan A, Nasrullah FD, Jaleel R (2016) Frequency and risk factors of low birth weight in term pregnancy. Pak J Med Sci 32(1): 138-142.

3. Black RE, Allen LH, Bhutta ZA, Caulfield LE, De Onis M, et al. (2008) Maternal and child undernutrition study group. Maternal and child undernutrition: Global and regional exposures and health consequences. The lancet 371(9608): 243-260.

4. Jeschke E, Biermann A, Günster C, Böhler T, Heller G, et al. (2016) Mortality and major morbidity of very-low-birth-weight infants in Germany 2008-2012: A report based on administrative data. Front Pediatr 4: 23.

5. Thompson JM, Wall C, Becroft DM, Robinson E, Wild CJ, et al. (2010) Maternal dietary patterns in pregnancy and the association with smallfor-gestational-age infants. Br J Nutr 103(11): 1665-1673.

6. Groce N, Challenger E, Bieler BR, Farkas A, Yilmaz N, et al. (2014) Malnutrition and disability: Unexplored opportunities for collaboration. J Paediatr Child Health 34(4): 308-314.

7. Risnes KR, Vatten LJ, Baker JL, Jameson K, Sovio U, et al. (2011) Birthweight and mortality in adulthood: A systematic review and metaanalysis. Int J Epidemiol 40(3): 47-661.

8. Rasmussen KM, Abrams B, Bodnar LM, Butte NF, Catalano PM, et al (2010) Recommendations for weight gain during pregnancy in the context of the obesity epidemic. Am J Obstetr Gynecol 116(5): 11911195.

9. Lee SE, Talegawkar SA, Merialdi M, Caulfield LE (2013) Dietary intakes of women during pregnancy in low-and middle-income countries. Public Health Nutr16(8): 1340-1353.

10. Abu Saad K, Fraser D (2010) Maternal nutrition and birth outcomes. Epidemiol Rev 32: 5-25.

11. Arimond M, Wiesmann D, Becquey E, Carriquiry A, Daniels MC, et al. (2010) Simple food group diversity indicators predict micronutrient adequacy of women's diets in 5 diverse, resource-poor settings. J Nutr 140(11): 2059-2069.

12. Boylan S, Lallukka T, Lahelma E, Pikhart H, Malyutina S, et al. (2011) Socio-economic circumstances and food habits in Eastern, Central and Western European populations. Public Health Nutr 14(4): 678-687.

13. Huddy RL, Torres SJ, Milte CM, McNaughton SA, Teychenne M, et al. (2016) Higher adherence to the Australian dietary guidelines is associated with better mental health status among Australian adult first-time mothers. J Acad Nutr Diet 116(9): 1406-1412.

14. Kennedy G, Ballard T, Dop M (2011) Guidelines for measuring household and individual dietary diversity. Food and Agriculture Organization. Rome, Italy.

15. Ali F, Thaver I, Khan SA (2014) Assessment of dietary diversity and nutritional status of pregnant women in Islamabad, Pakistan. J Ayub Med Coll Abbottabad 26(4): 506-509.

16. Saaka M (2013) Maternal dietary diversity and infant outcome of pregnant women in Northern Ghana. Int J Child Health Nutr 1(2): 148156.

17. Rahman A, Vahter M, Smith AH, Nermell B, Yunus M, et al. (2008) Arsenic exposure during pregnancy and size at birth: A prospective cohort study in Bangladesh. Am J Epidemiol 169(3): 304-312.

18. Saeed A, Raana T, Saeed AM, Humayun A (2015) Effect of antenatal depression on maternal dietary intake and neonatal outcome: A prospective cohort. J Nutr 15(1): 64. 
19. Ota E, Haruna M, Suzuki M, Anh DD, Tho LH, et al. (2011) Maternal body mass index and gestational weight gain and their association with perinatal outcomes in Viet Nam. Bull World Health Organ 89(2): 127136.

20. Sabour H, Nezhad HA, Maghbooli Z, Madani F, Mir E, et al. (2006) Relationship between pregnancy outcomes and maternal vitamin D and calcium intake: A cross-sectional study. Gynecol Endocrinol 22(10): 585-589.

21. Dasgupta A, Butt A, Saha TK, Basu G, Chattopadhyay A, et al. (2010) Assessment of malnutrition among adolescents: Can BMI be replaced by MUAC. Indian J Community Med 35(2): 276-279.

22. Vintzileos AM, Campbell WA, Rodis JF, Koefoed BR, Nochimson DJ (1987) Fetal weight estimation formulas with head, abdominal, femur and thigh circumference measurements. Am J of obstetr Gynecol 157(2): 410-414.

23. Procter KL, Rudolf MC, Feltbower RG, Levine R, Connor A, et al. (2008) Measuring the school impact on child obesity. Soc Sci Med 67(2): 341349.

24. Godfrey K, Robinson S, Barker DJP, Osmond C, Cox V (1996) Maternal nutrition in early and late pregnancy in relation to placental and fetal growth. BMJ 312(7028): 410.

25. Mikolajczyk RT, Ansari W, Maxwell AE (2009) Food consumption frequency and perceived stress and depressive symptoms among students in three European countries. J Nutr 8(1): 31.

26. Korkalo L, Erkkola M, Heinonen AE, Freese R, Selvester K, et al. (2017) Associations of dietary diversity scores and micronutrient status in adolescent Mozambican girls. Eur J Nutr 56(3): 1179-1189.
27. Ngala SA (2015) Evaluation of dietary diversity scores to assess nutrient adequacy among rural Kenyan women. Wageningen University, Netherlands.

28. Sibhatu KT, Krishna VV, Qaim M (2015) Production diversity and dietary diversity in smallholder farm households. Proc Natl Acad Sci USA 112(34): 10657-10662.

29. Savy M, Prevel MY, Danel P, Traissac P, Dabiré H, et al. (2008) Are dietary diversity scores related to the socio-economic and anthropometric status of women living in an urban area in Burkina Faso. Public Health Nutri 11(2): 132-141.

30. Thame M, Osmond C, Trotman H (2015) Fetal growth and birth size is associated with maternal anthropometry and body composition. Matern Child Nutr 11(4): 574-582.

31. Siyoum M, Mulaw Z, Abuhay M Kebebe H (2017) Implanon discontinuation rate and associated factors among women who ever used implanon in the last three years in Debre Markos town, Northwest Ethiopia, 2016, cross sectional study. Int J Community Med Public Health 2(1): 8-16.

32. Rammohan A, Goli S, Singh D, Ganguly D, Singh U (2019) Maternal dietary diversity and odds of low birth weight: Empirical findings from India. Women \& health 59(4): 375-390.

33. Zerfu TA, Umeta M, Baye K (2016) Dietary diversity during pregnancy is associated with reduced risk of maternal anemia, preterm delivery, and low birth weight in a prospective cohort study in rural Ethiopia. Am J Clin Nutr 103(6): 1482-1488. 